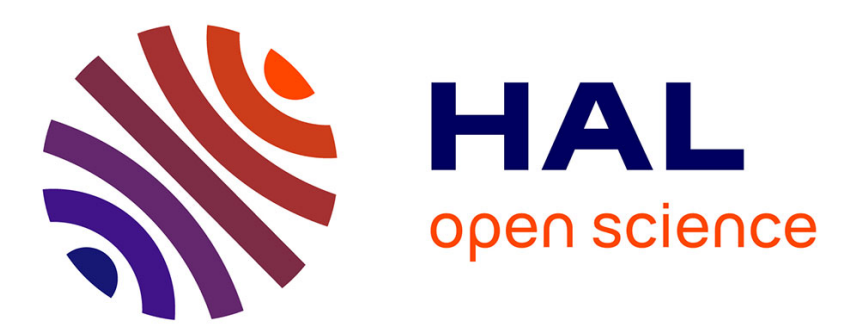

\title{
Hysteresis and vibration compensation in a NonLinear Unimorph Piezocantilever.
}

Micky Rakotondrabe, Cédric Clévy, Philippe Lutz

\section{To cite this version:}

Micky Rakotondrabe, Cédric Clévy, Philippe Lutz. Hysteresis and vibration compensation in a NonLinear Unimorph Piezocantilever.. IEEE/RSJ International Conference on Intelligent Robots and Systems, IROS'08., Sep 2008, Nice, France. pp.558-563. hal-00325162

\section{HAL Id: hal-00325162 \\ https://hal.science/hal-00325162}

Submitted on 26 Sep 2008

HAL is a multi-disciplinary open access archive for the deposit and dissemination of scientific research documents, whether they are published or not. The documents may come from teaching and research institutions in France or abroad, or from public or private research centers.
L'archive ouverte pluridisciplinaire HAL, est destinée au dépôt et à la diffusion de documents scientifiques de niveau recherche, publiés ou non, émanant des établissements d'enseignement et de recherche français ou étrangers, des laboratoires publics ou privés. 


\title{
Hysteresis and Vibration Compensation in a NonLinear Unimorph Piezocantilever
}

\author{
Micky Rakotondrabe, Cédric Clévy and Philippe Lutz
}

\begin{abstract}
Due to their rapidity and their high resolution, piezoelectric materials are very prized in microactuators and microrobotics. The classical example is the piezocantilevers. Notwithstanding, piezoecantilevers present nonlinearities (hysteresis and creep) when the applied electric field becomes large. On the other hand, they present lightly damped vibration. Feedback control is a classical issue to eliminate this unwanted behavior but it involves the use of sensors. In micromanipulation and in microassembly, sensors still remain one of the problematic because of their sizes and difficulty of packaging.

This paper presents the feedforward compensation of the hysteresis and the vibrations in piezocantilevers. While the Prandtl-Ishlinskii (PI) static hysteresis model is used to compute the hysteresis compensator, we employ the Input-Shaping method to reject the unwanted vibration. The experiments show that the accuracy can be highly increased while the setling time ameliorated and the vibration largely decreased.
\end{abstract}

\section{IntRoduCtion}

In the last decade, microrobotics have known a great interest notably in the field of micromanipulation and micro-assembly (especially for automated applications). In fact, these applications require very high performances in terms of positioning accuracy and repeatability that only microrobots could provide.

Microrobot are rarely based on hinges mechanisms because of the inaccuracy provided by the hinges. So, active materials based bending structures are commonly used to develop microactuators for these microrobots. However, active materials provide nonlinearities when the strain (and then the obtained displacement) is large. In addition, the performances obtained with these materials may vary according to the environmental condition variations [14]. Finally the model parameters may also vary according to the latter.

Closed loop control techniques seems to be the best way to reach substantial overall performances (accuracy, repeatability, disturbances rejection, etc.) for such problems. They notably enable a robust control that takes into account the uncertainties or variations of models parameters [11]. However, the use of closed loop control techniques in micromanipulation/microassembly is limited by the size of the sensors used: fast and precise

FEMTO-ST Inst., UMR CNRS 6174 - UFC / ENSMM / UTBM Automatic Control and Micro-Mechatronic Systems depart., (AS2M department)

25000 Besançon - France

\{mrakoton, cclevy, plutz\}@femto-st.fr enough sensors are bulky (interferometer, triangulation optical sensors, etc.) or difficult to fabricate [13]. Conversely, several studies demonstrated that open loop control techniques can also be an attractive approach: good satisfaction of overall performances for a very compact device [1][4][5].

This paper focuses on the open-loop control of a unimorph piezoelectric cantilever that will be integrated in a micromanipulation/microassembly system with force feeback. The cantilever is used at the same time as a manipulation actuator and a force measurement. A unimorph material has been chosen because of its elastic constant (around $2\left[\frac{\mu m}{m N}\right]$ for a PZT-Copper with a total size of $15 \mathrm{~mm} \times 2 \mathrm{~mm} \times 0.3 \mathrm{~mm})$ and because of the range of the manipulation force that we want to measure $( \pm 5 m N)$. While the force estimation technique was presented in a previous work [16], this paper presents the improvement of the performances of the piezocantilever with open-loop techniques.

On the one hand, cantilever structures are commonly used in the design of microrobotic devices. In fact, build with an active material, such structures replace the function of a hinged mechanism. On the other hand, piezoelectric materials are the most used due to their advantages: they can be used as sensor or actuator, their resolution is nearly unlimited (in the range of nanometers) and they have a fast dynamic. Withal, while piezoelectric materials present nonlinearities with large electric fields, bending structures (cantilevers) present badly damped vibrations. The piezoelectric nonlinearities are mainly the hysteresis and the creep. As the amplitude of the creep is very low (inferior to $0.25 \%$ of the final amplitude) for unimorph piezoelectric cantilevers when the environmental temperature variation is null [14], we only consider in this paper the compensation of the hysteresis and of the vibration.

Hysteresis influences a lot on the positioning repeatability. The hysteresis can be compensated with open loop control techniques, the goal being to obtain a global linear system. The computation of the compensator requires a precise model of the hysteresis. Several methods can be used, among them the most used are based on Preisach, Prandtl-Ishlinski, Bouc-Wen formulation or polynomial models [6] [8][10]. The Prandtl-Ishlinski is notably appreciated for its simplicity of implementation, ease of obtaining the inverse analytical formulation and its accuracy. For these reasons, this model will be used to compensate the hysteresis of the unimorph piezocantilever. 
The second important phenomenon is vibrations. They critically influences overshot of micromanipulation forces. This problem can be solved either by inverting the dynamic part of the model [2][3] or by choosing the shape of the input signal (input shaping) of the unimorph to minimize unwanted dynamics. When the dynamic model can be approached by a second order model, the latter is well adapted. On the other hand, the former is contrained by the use of bistable dynamic model (the model and its inverse must be stable). Input shaping [17] is a widespread technique for badly damped oscillating systems. A panorama of extended input shaping methods can be found in [18]. Due to the difficulty of integrating a sensor in micromanipulation systems, input shaping is a good approach to control their transient parts [5][13]. This method is used to reject the unwanted dynamic of the unimorph piezocantilever studied in this paper.

In section-II, static and dynamic characteristics of the unimorph are presented. The behaviour of the studied device is nonlinear mainly due to hysteresis and its dynamic is a badly damped vibration. Thus, while the section-III presents the implementation of a hysteresis compensator based on the Prandtl-Ishlinski model, the section-IV presents the input shaping control to cancel the vibrations.

\section{Characterization of the Piezocantilever}

In this section, we characterize the piezocantilever used for the experiments. We use a unimorph piezocantilever because it necessitates a low voltage to obtain large deflection. On the other hand, its characteristics, i.e. elastic constant and range of deflection, are suitable for our application: micromanipulation/microassembly actuator and force measurement $( \pm 5 m N)$. The piezoactuator is made up of a PZT layer and a Copper layer. The whole sizes are $15 \mathrm{~mm} \times 2 \mathrm{~mm} \times 0.3 \mathrm{~mm}$ (length, width and thickness). Fig. 1 presents the experimental setup. When a voltage is applied to the piezolayer, it expands or contracts. As the passive (Copper) layer is glued to it, the whole cantilever will bend.

A computer-DSpace board and an amplifier are used to supply the voltage and to treat the data from the sensor. The sensor is a Keyence laser sensor with $10 \mathrm{~nm}$ of resolution and some hundreds of nanometers of accuracy. The sensor is only used for the analysis. It is clear that the aim of the paper is to improve the performances of piezocantilevers without using sensors.

\section{A. Dynamic part analysis}

First, the transient part is analyzed. For that, we apply a step voltage with $40 \mathrm{~V}$ of amplitude to the piezocantilever. The result (Fig. 2) shows that the deflection $d$ response has a lightly damped vibration. This vibration may lead to fatal effect in some application cases. As example, the use of a microgripper where the two piezocantilevers have undamped vibration may damage the micro-object due to the overshoot of the force. Therefore, it is important to control this unwanted dynamic.

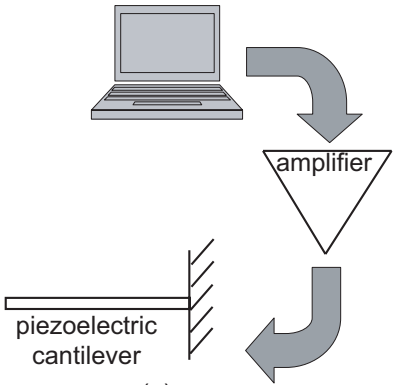

(a)

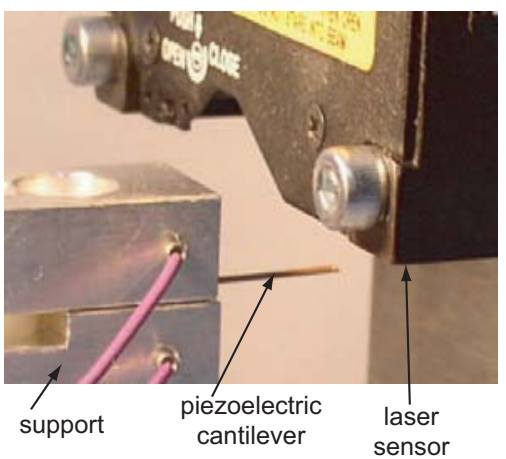

(b)

Fig. 1. The experimental setup.

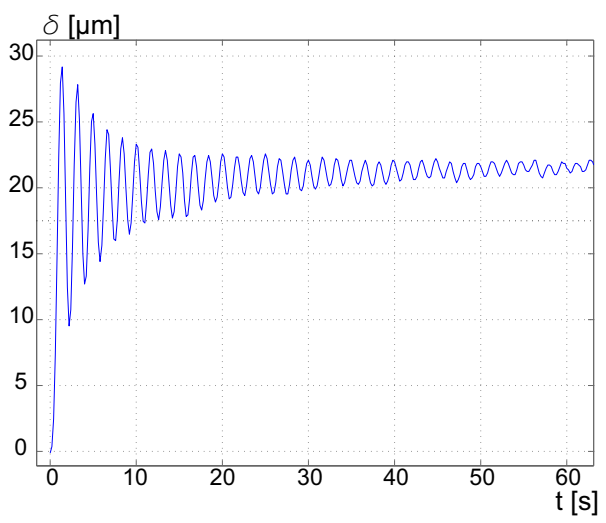

Fig. 2. Experimental step response $(U=40 \mathrm{~V})$.

\section{B. Static part analysis}

Second, we analyze the static plane $(U, \delta)$. For that we apply a sine voltage with an amplitude of $40 \mathrm{~V}$ and different values of frequency to the piezocantilever. The Fig. 3 show the results. The curves show that the behavior of the piezocantilever is hysteretic. Moreover, the hysteresis is dynamic (rate-dependent) because its shape depends on the frequency of the input voltage. Hysteresis phenomenon decreases the repeatability and the accuracy of a system. In micromanipulation/microassembly where the required accuracy is very high, the effect of hysteresis phenomenon must be reduced or eliminated. A dynamic hysteresis can be modeled and compensated using the Preisach dynamic model [12]. However a dynamic hysteresis $\Gamma_{d}(U, s)$, where $s$ is the Laplace variable, is equivalent to a static hysteresis $\Gamma(U)$ in series with a 


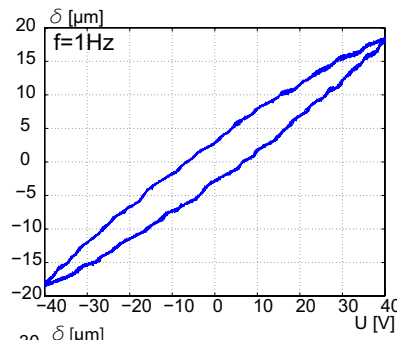

$30 \delta[\mathrm{mm}]$
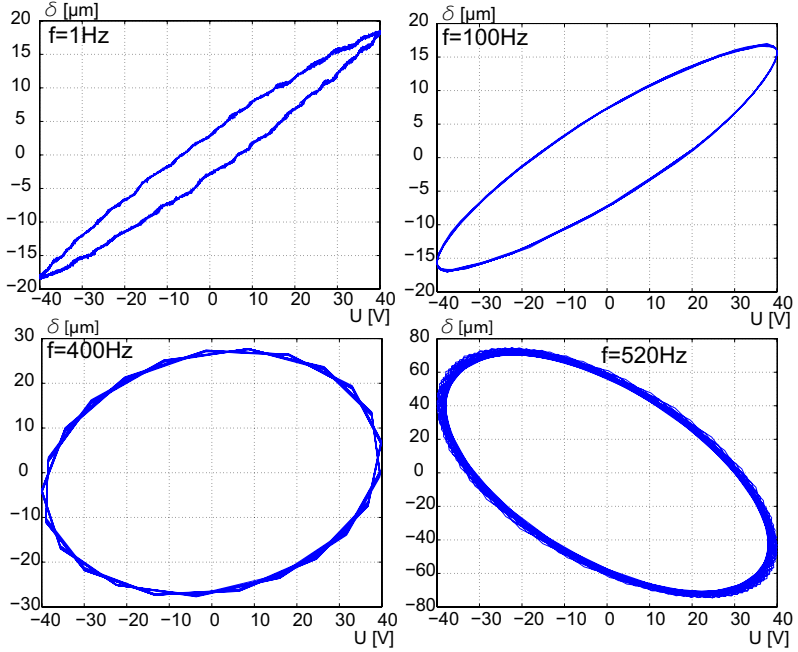

Fig. 3. Experimental results on the dynamic hysteresis behavior.

dynamic part $D(s)$ for piezoelectric cantilevers (example in $[3][15]$ ) (Fig. 4). We use this principle.
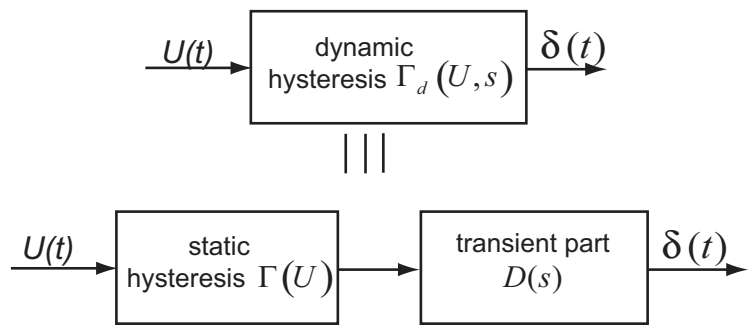

Fig. 4. Equivalence of a dynamic hysteresis and a static one in series with a dynamic part.

\section{Identification of the dynamic part}

To identify the dynamic part $D(s)$, the step response is used because the hysteresis only affects its static gain. The identified model is chosen to be a second order because we will compensate it with the input shaping method in the following sections. The Fig. 5 represents the step response of $D(s)$ and the experimental result as given in the Fig. 2. The static gain of $D(s)$ has been scaled in the figure in order to clearly show the comparison between both. We have chosen an order equal 2 because the computation will be based on such model:

$$
D(s)=\frac{1}{\frac{1}{\omega_{n}^{2}} \cdot p^{2}+\frac{2 . \xi}{\omega_{n}} \cdot p+1}
$$

where $\omega_{n}=3092 \mathrm{rad} / \mathrm{s}$ is the natural frequency and $\xi=0.029$ is the damping ratio. In the following sections, the system is first linearized by compensating the static hysteresis. After that, the unwanted vibration in $D(s)$ is eliminated.

\section{Hysteresis COMPENSATION}

The feedforward hysteresis compensation first needs a precise model $\Gamma(U)$. To perform the linearization,

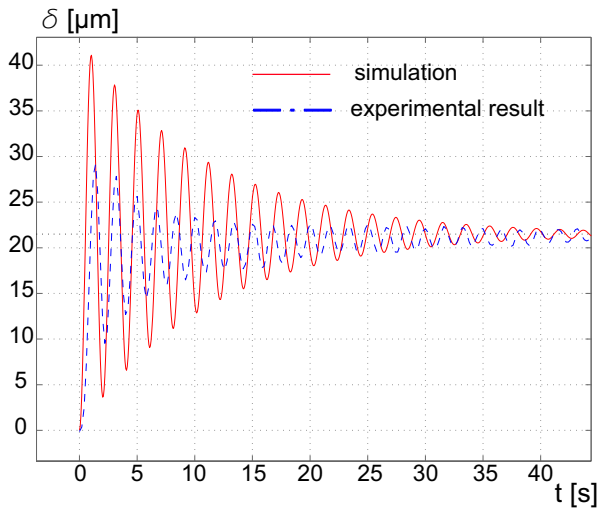

Fig. 5. Simulation of $D(s)$ (with a scaled gain) compared with the experimental result.

the inverse model $\Gamma^{-1}(U)$ is put in cascade with the system. We have chosen the Prandtl-Ishlinskii (PI) model because of its simplicity and accuracy.

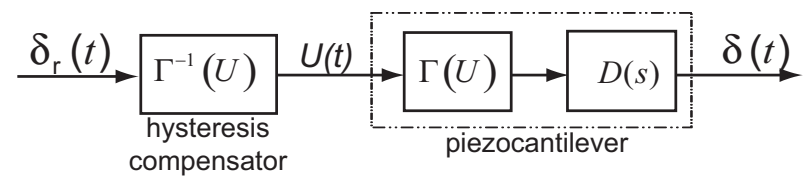

Fig. 6. Scheme of the hysteresis compensation.

\section{A. The Prandtl-Ishlinskii (PI) static hysteresis}

The PI model is based on the superposition of many elementary backlash operators characterized by the threshold $r$ and with weighting coefficient $h$ (Fig. 7) [9]. Let

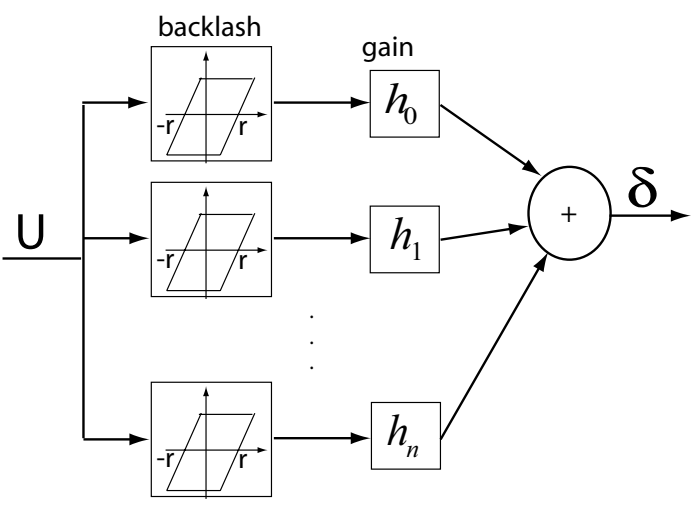

Fig. 7. The PI static hysteresis model.

$H_{r i}\left[U, \delta_{O i}\right](t)$ be the $i$-th backlash operator characterized by its threshold $r i$ and the initial state $\delta_{O i}$. The complex hysteresis is given by the following equation:

$$
\delta=\Gamma(U)=[h]^{T} \cdot\left[H_{r}\left[U, \delta_{O}\right](t)\right]
$$

where:

- $\left[H_{r}\left[U, \delta_{O}\right](t)\right]=\left[\begin{array}{lll}H_{r 0}\left[U, \delta_{O 0}\right](t) & \ldots & H_{r n}\left[U, \delta_{O n}\right](t)\end{array}\right]^{T}$ is the vector of backlash operators,

- $[h]=\left[\begin{array}{lll}h_{0} & \ldots & h_{n}\end{array}\right]^{T}$ is the weight vector, 
- and $n+1$ is the number of backlash operators.

To compute the inverse PI model, another static hysteresis is considered. In this, while the deflection $\delta$ is the input, the voltage $U$ is the output. The same principle than the direct PI model can be applied. The new thresholds $r_{i}^{\prime}$ and weighting elements $h_{i}^{\prime}$ are given as follow [8]:

$$
r_{i}^{\prime}=\sum_{j=0}^{i} h_{j} \cdot\left(r_{i}-r_{j}\right) \quad ; \quad i=0 \ldots n
$$

and

$$
\begin{aligned}
h_{0}^{\prime} & =\frac{-1}{h_{0}} \\
h_{i}^{\prime} & =\frac{-h_{i}}{\left(h_{0}+\sum_{j=1}^{i} h_{j}\right)\left(h_{0}+\sum_{j=1}^{i-1} h_{j}\right)} \quad ; \quad i=1 \ldots n
\end{aligned}
$$

\section{B. Experiments}

The PI model has been identified with 15 backlash operators. For that, a sine voltage with $40 \mathrm{~V}$ of amplitude is applied to the piezocantilever. The frequency is chosen to be $1 \mathrm{~Hz}$. To avoid the effect of the dynamic on the hysteresis shape, the frequency must not be higher than $400 \mathrm{~Hz}$. On the other hand, it must not be lower than $5 \mathrm{mHz}$ to avoid the creep effect.

After identification, the inverse model (compensator) is computed. The compensator has been implemented in the Matlab-Simulink software and a computer-DSpace hardware. The reference $\delta_{r}(t)$ is a sine signal with the same frequency and two different values of amplitude $(7.5 \mu \mathrm{m}$ and $15 \mu \mathrm{m})$. These amplitudes respectively correspond to nearly $20 \mathrm{~V}$ and $40 \mathrm{~V}$. Indeed, in the PI model the range of the purpose voltage must be inferior to the range of the identification voltage.

The results are interesting (Fig. 8). The accuracy between the output deflection $\delta$ and the input reference $\delta_{r}$ is high. The two curves coincide themselves and no residual hysteresis is presented. In order to see the linearity, the

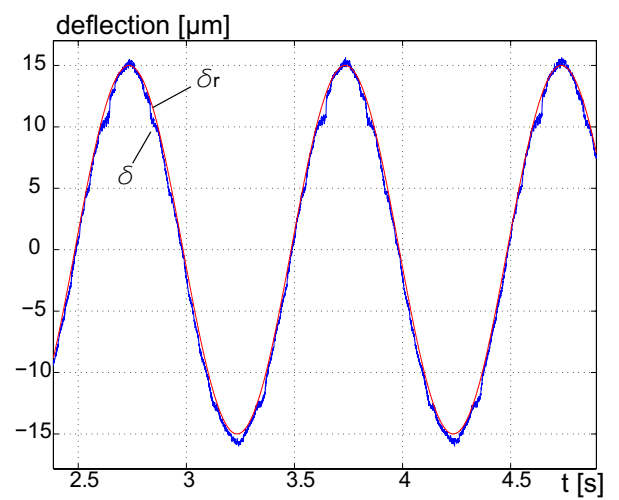

Fig. 8. Hysteresis compensation: experimental results with a sine input reference $\delta_{r}(t)$ with $1 H z$ frequency and $15 \mu m$ of amplitude.

curves were plotted in the $(U, \delta)$-plane. Fig. 9 show the results with different amplitudes and frequency. While the hysteresis was initially nearly $18.6 \%$ (see Fig. 4 at $f=$
$1 \mathrm{~Hz}$ ), it was completely eliminated in the compensated results. From the figures, it can be deduced that the linear gain between the output $\delta$ and the reference $\delta_{r}$ is equal to one.
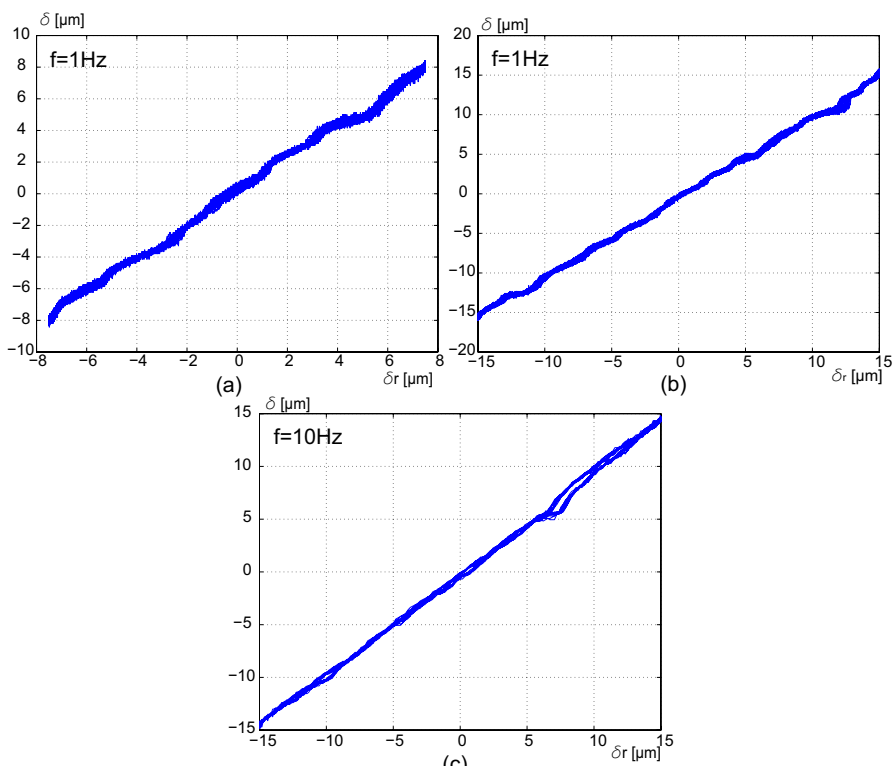

Fig. 9. Hysteresis compensation: experimental results with a sine input reference $\delta_{r}(t)$ of different amplitude and frequency.

\section{VIBRATION COMPENSATION}

The new system, i.e. hysteresis compensator and piezocantilever, is now linear but always contains unwanted dynamic. In this section, we compensate the vibration using the classical shaping technique also called ZV (Zero Vibrations) input shaping technique. The technique is first presented. Afterwards the experimental results are given.

\section{A. The input shaping technique}

There are different kind of input shaping methods but the one presented here is the Zero Vibration (ZV) technique. A panorama of other existing methods is given in [18]. The ZV input shaping method [17] is a simple technique to minimize the vibration in lightly damped systems. It is based on the convolution of a sequence of impulses called shaper and an input signal.

When an impulse is applied to an oscillating system, a vibration appears. Let $\omega_{n}$ be the natural frequency and $\xi$ the damping ratio. When a second impulse is applied at time $T_{d}=T / 2$, with $T=\frac{2 . \pi}{\omega_{n} \cdot \sqrt{1-\xi^{2}}}$, the vibration caused by the second impulse can cancel the one caused by the first impulse (Fig. 10) if the amplitudes of both are judiciously chosen. To apply a reference input, the precedent sequence of impulses, also called shaper, is convolved with it to obtain a new signal control that will cause no vibration. As example, if the reference input is a step, the resulting signal control will be a staircase with two steps. The computation of the shaper is given as 


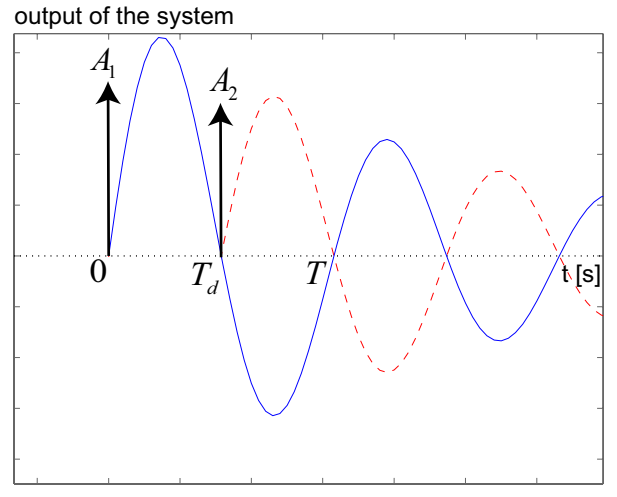

Fig. 10. Use of 2 impulses in an oscillating system.

follow. Consider $A_{i}$ and $t_{i}$ the amplitudes of the impulses and their application times. Consider $K=e^{-\frac{\xi \cdot \pi}{\sqrt{1-\xi^{2}}}}$. A shaper with two impulses has:

$$
\begin{aligned}
& {\left[\begin{array}{l}
\left.A_{1}=\frac{1}{1+K}, \quad A_{2}=\frac{K}{1+K}\right] \\
t_{1}=0, \quad t_{2}=T_{d}
\end{array}\right]}
\end{aligned}
$$

When residual vibration appears even due to an uncertainty on the used natural frequency or damping ratio, a higher number of impulses of the shaper is used. So, when a step reference input is applied, a staircase signal control with several steps is provided by the shaper to the system. For a shaper with $m$ impulses, we have:

$$
\begin{aligned}
& {\left[A_{1}=\frac{a_{1}}{(1+K)^{n-1}}, \quad A_{2}=\frac{a_{2}}{(1+K)^{n-1}},\right.} \\
& \left.\ldots, \quad A_{m}=\frac{a_{m}}{(1+K)^{m-1}}\right] \\
& {\left[t_{1}=0, \quad t_{2}=T_{d}, \quad \cdots, \quad t_{m}=(m-1) T_{d}\right]}
\end{aligned}
$$

with $a_{i}$ the $i^{\text {th }}$ coefficient of the polynomial from $(1+K)^{m-1}$. We have $a_{1}=1$ and $a_{m}=K^{m-1}$. Using the dynamic model given by the equation (1) and the identified parameters, a shaper has been computed. It has been implemented in cascade with the linearized system, i.e. the hysteresis compensator and the piezocantilever (Fig. 13). The Matlab-Simulink software and a computerDSpace setup were used to implement the two compensators.

In the Fig. 13, we have:

- $A_{i}$ as the amplitude of the impulses and $D_{i}$ the delays of the shaper,

- $r$, as the threshold and $h_{j}$ the gain of the hysteresis compensator.

First, we have performed experiments with a the step reference having $\delta_{r}= \pm 15 \mu m$ of amplitude. Shapers with different number of impulses were tested. The step responses indicated that the performances did not increase substantially when using impulses number greater than four. The Fig. 11 shows the experimental step response of the compensated system (shaper with $1,2,3$ and 4 impulses) and of the system without shaper. The Table I summarizes the performances. They point out that the overshoot has been reduced from $50.8 \%$ to $0 \%$ when the impulses number is four. In addition, a high reduction of the setling time is obtained. Finally, the results point out that there is no major amelioration of the performances from 3 impulses number. It is noticed that increasing the number of impulses also increases the action time of the shaper but it has been shown that it is still possible to shape the input without changing any specified time [7]. Next, we have performed a harmonic

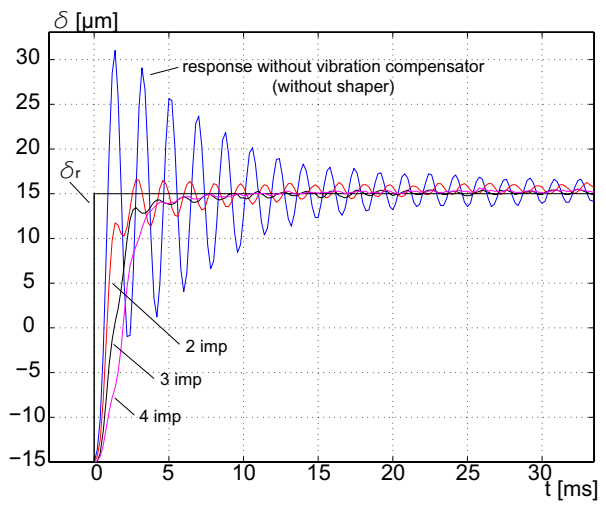

Fig. 11. Experimental results: step response.

analysis. The Fig. 12 presents the results. They show that not only the peak at the resonant frequency was reduced, but it becomes antiresonant. The more the impulses number is high, the more the antiresonant phenomenon is important.

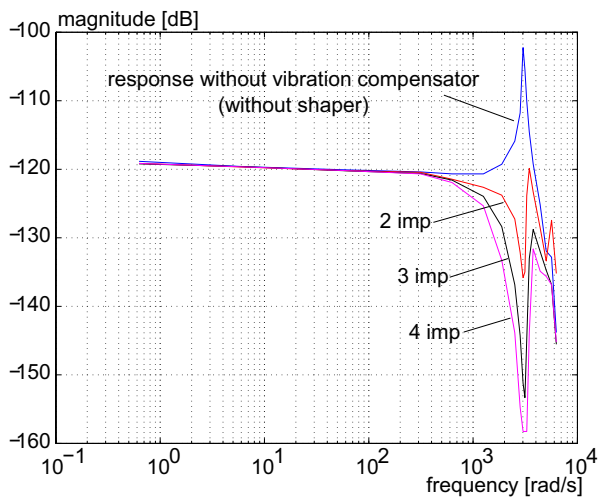

Fig. 12. Experimental results: harmonic response (magnitude).

\section{B. Experiments}

\section{Conclusion}

The use of feedforward control techniques are of great interest in micromanipulation/microassembly because they do not require sensors. Indeed, accurate sensors are nowadays spacious and are not convenient for downscaling manipulation systems.

This paper presents the compensation of the hysteresis and vibration in piezocantilevers using the feedforward control techniques. The hysteresis was first compensated 
TABLE I

Performances.

\begin{tabular}{|l||l||l|}
\hline \hline & overshoot & setling time \\
\hline uncompensated vibration & $50.8 \%$ & $>28.5 \mathrm{~ms}$ \\
\hline compensated vibration (2 impulses) & $3.65 \%$ & $10.7 \mathrm{~ms}$ \\
\hline compensated vibration (3 impulses) & $0 \%$ & $3.95 \mathrm{~ms}$ \\
\hline compensated vibration (4 impulses) & $0 \%$ & $\approx 4 \mathrm{~ms}$ \\
\hline
\end{tabular}



Fig. 13. Principle scheme of the hysteresis and vibration compensation.

in order to linearize the system. The Prandtl-Ishlinskii (PI) inverse model was used because of its ease of implementation and its accuracy. During the experiments, the implemented compensator has completely cancelled the initial hysteresis of $18.6 \%$ and has increased the accuracy of the positioning. Next, the vibration of the new system (piezocantilever and hysteresis compensator) was compensated. The input shaping method was used. Different number of impulses were tested for the shaper. The results were interesting: the shaper completely removes the overshoot and the setling time largely decreases relative to the one of the uncompensated system. Finally, the harmonic experiments have shown that the resonant peak has became an antiresonant phenomenon.

The experiments presented in the paper indicate that the performances of the unimorph piezocantilever controlled with open loop techniques correspond to those required in micromanipulation.

\section{ACKNOWLEDGMENT}

This work is partially supported by the European Project EUPASS (http://www.eupass.org/).

\section{REFERENCES}

[1] B. Borovic, A. Q. Liu, D. Popa, H. Cai, and F. L. Lewis. Openloop versus closed-loop control of mems devices: choices and issues. Journal of Micromech. and Microeng., 2005.

[2] G.M. Clayton, S. Tien, A.J. Fleming, S.O.R. Moheimani, and S. Devasia. Inverse-feedforward of charge-controlled piezopositioners. In Mechatronics, 2007.

[3] D. Croft, G. Shed, and S. Devasia. Creep, hysteresis, and vibration compensation for piezoactuators: Atomic force microscopy application. ASME Jour. of Dyn. Syst., Meas. and Control, 2001.

[4] M. Daqaq. Adaptation of nontraditional control techniques to nonlinear micro and macro mechanical systems. PhD thesis, PhD. Virginia Tech., 2006.
[5] S. Devasia, E. Eleftheriou, and S. O. R. Moheimani. A survey of control issues in nanopositioning. IEEE Trans. on Cont. Syst. Tech., 2007.

[6] A. Dubra, J. Massa, and C.l Paterson. Preisach classical and nonlinear modeling of hysteresis in piezoceramic deformable mirrors. Optics Express, 2005.

[7] S. Kapucu G. Alici and S. Bayseç. A robust technique for motion design of flexible-jointed robotic systems. 2006.

[8] K. Kuhnen and H. Janocha. Inverse feedforward controller for complex hysteretic nonlinearities in smart material systems. Control and Intelligent System, 2001.

[9] K. Kuhnen and H. Janocha. Complex hysteresis model of a broad class of hysteretic nonlinearities. In Conference on New Actuators, 2002.

[10] K. K. Leang. Iterative Learning Control of Hysteresis in Piezobased Nano-positioners: Theory and Application in Atomic Force Microscopes. PhD thesis, PhD. University of Washington, 2004.

[11] K. K. Leang and S. Devasia. Hysteresis, creep, and vibration compensation. In IFAC Conference on Mechatronic Systems, 2002.

[12] I. D. Mayergoyz. Mathematical models of hysteresis and their applications. Elsevier, 2003.

[13] D. O. Popa, B. H. Kang, J. T. Wen, H. E. Stephanou, G. Skidmore, and A. Geisberger. Dynamic modeling and input shaping of thermal bimorph mems actuators. In IEEE ICRA, 2003.

[14] M. Rakotondrabe, C. Clévy, and P. Lutz. H-inf deflection control of a unimorph piezoelectric cantilever under thermal disturbance. In IEEE ICRA, 2007.

[15] M. Rakotondrabe, Y. Haddab, and P. Lutz. Plurilinear modeling and discrete $\mu$-synthesis control of a hysteretic and creeped unimorph piezoelectric cantilever. In IEEE ICARCV, 2006.

[16] M. Rakotondrabe, Y. Haddab, and P. Lutz. Nonlinear modeling and estimation of force in a piezoelectric cantilever. In IEEE/ASME AIM, 2007.

[17] N. C. Singer, W. P. Seering, and K. A. Pasch. Shaping command inputs to minimize unwanted dynamics. In Patent $N \check{r} U S-4.916 .635,1990$.

[18] T. Singh and W. Singhose. Tutorial on input shaping/time delay control of maneuvering flexible structures. In American Control Conference, 2002. 